\title{
Las ideas sobre el desarrollo en la historia del pensamiento ambientalista latinoamericano
}

Aín Mora ${ }^{1}$, Guillermo Peinado ${ }^{2}$

\begin{abstract}
RESUMEN
El documento presente pretende abordar, de manera histórica, las diferentes ideas sobre la relación entre producción y medio ambiente a lo largo de la historia reciente en Latinoamérica. Específicamente, pretendemos elaborar como esas ideas entre producción y medio ambiente penetraron en la sociedad civil y cómo fue su relación en torno al concepto de desarrollo. El objetivo de este trabajo no es generar consenso sobre las definiciones y los principales aportes al pensamiento ambiental latinoamericano. Nuestra elaboración busca aportar una visión general y personal, cuya intención es marcar algunos ejes críticos que permitan un mayor desarrollo de este pensamiento en nuestra región. En relación con el objetivo general se verifica que el devenir histórico de las ideas ambientalistas derivó en dos corrientes antagónicas: por un lado, una propuesta asociada al desarrollo sustentable expresada por los organismos internacionales en donde existe una conciliación entre desarrollo y medio ambiente; al contrario de esta propuesta se presenta una idea más identitaria que señala las contradicciones entre el concepto de desarrollo y ambiente y propone la elaboración de prácticas económicas alternativas que se enfrentarse a las visiones tradicionales hegemónicas del desarrollo sustentable.
\end{abstract}

Palabras-clave: pensamiento ambientalista; ecodesarrollo; desarrollo sustentable; ecologismo popular.

\footnotetext{
1 Maestrando en Historia Social Argentina y Latinoamericana (UNR, Argentina), Licenciado en Economía (UNR, Argentina). Instituto de Investigaciones Económicas, Facultad de Ciencias Económicas y Estadística (UNR, Argentina). Miembro de la Asociación Argentino-Uruguaya de Economía Ecológica (ASAUEE). ORCID: https://orcid.org/0000-0002-8694-121X Email: amora@fcecon.unr.edu.ar

${ }^{2}$ Magíster en Economía Política (FLACSO, Argentina), Licenciado en Economía (UNR, Argentina). Instituto de Investigaciones Económicas, Facultad de Ciencias Económicas y Estadística (UNR, Argentina). Miembro de la Asociación Argentino-Uruguaya de Economía Ecológica. ORCID: https://orcid.org/0000-0003-1100-4296 E-mail: gpeinado@fcecon.unr.edu.ar
} 

antigua en América Latina tanto política como epistemológicamente. En este trabajo nos proponemos abordar esta relación desde el campo de las ideas que circulaban por los pensadores ambientales en nuestro continente. En relación con esta temática se analizará como esa construcción de "nuevos" conceptos influenció a la sociedad civil mediante la creación de asociaciones, organizaciones no gubernamentales (ONG's) y movimientos sociales que combatían las problemáticas socio-ambientales. Nos interesa, principalmente, la conexión entre algunas ideas surgidas a partir de la negación de la contradicción entre ambiente y desarrollo económico y las ideas latinoamericanas que aceptan esa contradicción y diagraman, sobre el territorio, nuevos parámetros para pensar los conflictos socioambientales.

En ese sentido, las ideas aquí recopiladas seguirán el esquema diagramado por Devés-Valdés en su libro "El Pensamiento latinoamericano en el siglo XX". Es decir, analizaremos las ideas ambientales desde la óptica de ciclos entre lo identitario y lo modernizador ${ }^{3}$.

Entendemos, como cita Devés Valdés, que este criterio de las tensiones entre modernización e identidad es válido para una gran parte del pensamiento latinoamericano pero que hay autores y sobre todo perspectivas que no se enmarcan en esta polaridad ${ }^{4}$. Hecha esta salvedad, entendemos que este tipo de análisis enriquece el panorama teórico en curso y puede aportar una nueva visión sobre el desarrollo y la relación producción-medio ambiente.

\footnotetext{
3"Definen al proyecto modernizador los siguientes énfasis: a) Afán de seguir el ejemplo de los países más desarrollados; b) Acentuación de lo tecnológico, de lo mecánico en desmedro de lo cultural, de lo artístico, de lo humanista; c) La convicción de que son los países más desarrollados 0 sus habitantes quienes pueden en mejor forma promover la modernización de nuestros países y por ello se propician formas de intervencionismo 0 de radicación de ciudadanos de dichos países para que importen con

ellos sus pautas culturales; d) Énfasis en ponerse al día; e) Énfasis en la apertura al mundo; f) Desprecio de lo popular, de lo indígena, de lo latino, de lo hispánico, de lo latinoamericano; g) Énfasis en la eficiencia, la productividad, en desmedro de la justicia y la igualdad. Caracterizan al proyecto identitario, por su parte, los énfasis siguientes: a) La reivindicación y defensa de lo americano, de lo latino, de lo indígena, de lo propio; b) La valoración de lo cultural, lo artístico, lo humanista en desmedro de lo tecnológico (sea por olvido o por desprecio); c) El no intervencionismo de los países más desarrollados en América latina, la reivindicación de la "independencia" y de la "liberación"; d) Acentuación de la justicia, de la igualdad, de la libertad; e) La reivindicación de una manera peculiar de ser, distinta a la de los países más desarrollados, en la cultura y en el tiempo propios; f) Énfasis en el encuentro consigo mismo, con el país, con el continente", Devés Valdés, Eduardo. «El pensamiento latinoamericano a comienzos del siglo XX: La reivindicación de la identidad.» CUYO, Anuario de Filosofía Argentina y Americana, N 14, 1997: 1175: pp. 14

${ }^{4}$ Devés Valdés, Eduardo. «El pensamiento latinoamericano a comienzos del siglo XX: La reivindicación de la identidad.» CUYO, Anuario de Filosofía Argentina y Americana, № 14, 1997: pp. 11-75.
} 
Vale aclarar que este ensayo no pretende ser un análisis que busque generar un consenso sobre las definiciones y los principales aportes al pensamiento ambiental latinoamericano. Tampoco busca incluir a todos los nombres de autores, organizaciones, textos o prácticas ambientalistas. Nuestra elaboración solo es un esbozo general y personal, cuya intención es marcar algunos ejes críticos que permitan un mayor desarrollo de este pensamiento en nuestra región. Por cuestiones de extensión se dejan por fuera algunos debates relacionados a la rama de las políticas de conservación ambiental.

Bajo ese objetivo dividiremos al mismo en tres partes diferenciadas. En una primera parte desarrollaremos el concepto de "Ecodesarrollo" que dio paso al auge del ciclo modernizador a través del concepto de "desarrollo sustentable". Específicamente, profundizaremos sobre cómo se originó el denominado "Ecodesarrollo" y como se transformó tanto en el ideario académico como en las prácticas gubernamentales en el concepto de "desarrollo sustentable" asociado a una relación armónica entre producción e impactos ambientales.

Por el contrario, en la segunda parte se especificará las diferentes teorías surgidas como una negación a la conciliación entre desarrollo y ambiente y en respuesta a la lógica extractivista y exportadora. Estas ideas surgieron en el seno del pensamiento latinoamericano teniendo en cuenta las problemáticas específicas de esta región por lo que marcan un ciclo identitario del pensamiento ambientalista.

Por último, se elaborará una conclusión sobre el pensamiento ambientalista latinoamericano y su devenir histórico en la actualidad.

\section{El proyecto modernizador: Del "Ecodesarrollo" al "Desarrollo Sustentable", del INTENTO DE HACER CAMINO AL CAMINO DE LOS OTROS.}

El tema del medio ambiente se terminó de instalar formalmente ${ }^{5}$ en la agenda política mundial tras la realización de la Conferencia sobre el Medio Humano, citada 
por la Organización de las Naciones Unidas y realizada en Estocolmo en 1972. En esta conferencia se creó el Programa de Naciones Unidas para el Medio Ambiente (PNUMA) y se caracterizó a la crisis reinante como el resultado del modo de vida industrial llevado a cabo en estos países. La resolución de estos problemas, según se podía leer en la Declaración Final de la Conferencia, requería de una amplia colaboración entre las naciones del mundo ${ }^{6}$.

La iniciativa de dicha conferencia fue llevada a cabo por los países altamente industrializados, quienes tenían una perspectiva distinta de la cuestión ambiental en comparación de los países categorizados como "en vías de desarrollo". Por un lado, los países industrializados se centraban en las variables de contaminación, polución industrial y el crecimiento demográfico del Tercer Mundo. Este último problema es el que va a estar en los debates del Club de Roma, creado en 1968, y el que guía el conocido informe titulado "Los Límites del Crecimiento" publicado en 1972. Por otro lado, las preocupaciones de los países de nuestro continente giraban en otras direcciones, mucho más cercanas a los problemas reales latinoamericanos, no vinculados a problemas industriales sino a problemas que tenían que ver con la pobreza, la desigualdad y el daño ambiental surgido por una estructura económica distinta a la de los países del "primer mundo". Como escribiría Guimaraes tiempo después de la Conferencia de Río: "No debería sorprender el alto grado de resistencia demostrado por los países del Tercer Mundo en aquel entonces. Como lo resumió el representante del gobierno de la India en una reunión preparatoria a Estocolmo llevada a cabo en Puerto Rico: Los ricos se preocupan del humo que sale de sus autos; a nosotros nos preocupa el hambre"7

En el marco del pensamiento ambiental, la visión desarrollista y la agenda marcada de los países hegemónicos fue contrastada, en parte, por una visión que se anunciaba como más latinoamericana. En este sentido, la respuesta a la crisis ambiental global encontrará en nuestro continente la difusión de las llamadas "estrategias del

Sejenovich, H, B Slutsky, y S Cabrera. Rescatando la historia perdida. El pensamiento ambiental latinoamericano a la luz de las contradicciones actuales del desarrollo. Buenos Aires: ENGOV, 2012.

${ }^{6}$ Estenssoro Saavedra, Fernando. «El Ecodesarrollo como concepto precursor del desarrollo sustentable y su influencia en América Latina.» Universum, Universidad de Talca. Vol. 30. № 1, 2015: pp. 81-99

7 Guimaraes, Roberto. «El discreto encanto de la cumbre de la tierra. Evaluación impresionista de Río-92.» Nueva Sociedad N¹22, 1992: 86-103. 
Ecodesarrollo", las cuales abogaban por la necesidad de crear nuevas formas de producción y estilos de vida basados en las condiciones y potencialidades ecológicas de cada región, así como en la diversidad étnica y la capacidad de las poblaciones locales para la gestión participativa de los recursos ${ }^{8}$.

Esta visión del Ecodesarrollo está en línea con las discusiones de la época. En la década de 1950 se había consolidado la economía del desarrollo que tenía como objeto de estudio principal las denominadas "economías subdesarrolladas". A partir de los procesos surgidos en la segunda posguerra bajo el esquema teórico keynesiano y con las nuevas tecnologías para el manejo social, la preocupación clásica por la acumulación de capital se convirtió en el núcleo de los primeros intentos por aplicar a los países "subdesarrollados" las herramientas del análisis económico. "El énfasis central estaba puesto en la inversión extranjera ya que se suponía que los países subdesarrollados no tenían suficiente capital para satisfacer las inversiones requeridas." En este sentido, esta economía del desarrollo argumentaba que el campo privilegiado para esas inversiones era la industrialización, cuyos beneficios garantizaban la vía para la modernización de estas economías atrasadas ${ }^{9}$. Esta teoría de consolidó en la década de 1960, con el compromiso de la mayoría de los gobiernos latinoamericanos con la planeación, la reforma agraria y la Alianza para el Progreso.

En este contexto histórico, el concepto de Ecodesarrollo fue explicado en profundidad por un economista polaco, naturalizado posteriormente brasileño, Ignacy Sachs. Sachs publicó sus planteamientos en el artículo "Ambientes y estilos de desarrollo" en 1974. El autor señalaba que era posible un desarrollo social continuo en armonía con el medio ambiente, pero para esto era necesario entender que el ambiente

\footnotetext{
${ }^{8}$ Leff, Enrique. «El retorno del orden simbólico: la capitalización de la naturaleza y las estrategias fatales del desarrollo sostenible.» En La racionalidad ambiental, de Enrique Leff, 88-132. México D.F.: Siglo XXI, 2004.

${ }_{9}^{9}$ Podemos citar dos teorías muy influyentes que le daban sustento a estas prácticas. La primera es el modelo de William Arthur Lewis de la economía dual (1954). El núcleo del modelo formula que los países subdesarrollados tenían dos sectores que convivían entre sí: uno moderno y otro tradicional. El desarrollo económico apuntaba a la invasión progresiva del sector tradicional por parte del moderno y en la expansión sostenida de la economía monetaria sobre la economía de subsistencia. Esto se traduciría en la movilización del subempleo rural (de baja productividad marginal) hacia zonas urbanas donde son contratados por las nuevas industrias con salarios de subsistencia (mayores que el sector rural) lo que crearía un mayor ahorro adicional.

El segundo modelo teórico era el de Raúl Prebisch (1950), economista argentino que en conjunto con la Comisión Económica para América Latina (CEPAL) demostró que la causa del atraso era el deterioro histórico de los términos de intercambio que afectaba a los bienes primarios de los países de la periferia. Este deterioro era producido por la concentración de los adelantos del progreso técnico en el centro industrializado y derivaba en un producto por trabajador menor en la periferia. Dado el excedente de mano de obra que existía en estos países, la conclusión de Prebisch es que los países periféricos tenían una menor capacidad de acumulación de capital y, por lo tanto, se necesitaba políticas estatales de industrialización que permitieran a los países producir en casa los bienes que antes importaban.
} 
era una dimensión del desarrollo y por esto debía ser asimilado en todos los niveles de decisión, lo que obligaba a replantearse las definiciones de desarrollo y entender que podían existir múltiples formas del mismo. Específicamente, en cuestiones del "desarrollo tercermundista" y los ecosistemas tropicales, Sachs propuso que el Ecodesarrollo era una forma de desarrollo adaptado a las realidades eco-sistémicas de cada región o eco-región.

Si bien el autor no proponía salir del camino del desarrollo, criticaba la idea catastrófica que los países dominantes tenían respecto del crecimiento demográfico de los países "subdesarrollados". El objetivo de Sachs no era el de detener el desarrollo sino el de reorientarlo en una perspectiva ecológica y sana ambientalmente. En este sentido, el Ecodesarrollo buscaba un proceso de "desarrollo humanista" consciente de $\mathrm{su}$ interdependencia con la naturaleza y, para lo cual postulaba colocar el avance científico-técnico al servicio de la protección de los ecosistemas, o ecotécnica ${ }^{10}$.

Este concepto expuesto por Sachs resonó en el incipiente pensamiento ambiental latinoamericano. Sachs influenció a Enrique Leff, quien fuera su discípulo en la École des Hautes Études en Sciences Sociales. En enero de 1975, Leff publicó, "Hacia un proyecto de Ecodesarrollo", para plantear la necesidad de aplicar en países latinoamericanos y del Tercer Mundo estilos de desarrollo que estuvieran de acuerdo con sus realidades físicas que condicionan la naturaleza y los recursos, así como sus realidades socio-culturales e históricas, ya que de esta forma se podían tener mejores rendimientos energéticos y productivos asegurando un desarrollo a largo plazo, y esto era mucho mejor que la trasplantación mecánica de los estilos de desarrollo primermundistas unilineales que se transformaban en malas imitaciones de lo que ocurría en los países desarrollados ${ }^{11}$.

La promoción del Ecodesarrollo en nuestra región tuvo su apogeo entre 1972 y 1982, entre la Conferencia de Estocolmo sobre el Medio Ambiente Humano y el comienzo de la crisis de la deuda en los países latinoamericanos. Intelectuales y científicos latinoamericanos estaban vinculados principalmente a la Comisión

\footnotetext{
10 Estenssoro Saavedra, Fernando. «El ecodesarrollo como concepto precursor del desarrollo sustentable y su influencia en América Latina.» Universum, Universidad de Talca. Vol. 30. No 1, 2015: 81-99.

11 Estenssoro Saavedra, Fernando. Historia del debate ambiental en la política mundial (1945-1992). Santiago de Chile: Instituto de Estudios Avanzados. Universidad Santiago de Chile. , 2014.
} 
Económica para América Latina (CEPAL) y al Programa de las Naciones Unidas para el Medio Ambiente (PNUMA) y a institutos dedicados al estudio de los recursos naturales y a la problemática medio ambiental de sus países. Una de las referencias claves en este sentido fue el proyecto de la CEPAL denominado "Estilos de desarrollo y medio ambiente en la América Latina" (1978-1980) que marcó las líneas de estudios y de asesorías a los países de la región y durante veinte años contribuyó a la conceptualización de la relación desarrollo y medio ambiente ${ }^{12}$. Estos análisis se mantienen hasta el día de hoy y se resaltan autores como Nicolás Giglo y Osvaldo Sunkel.

El Ecodesarrollo habría de ser el principal motor que llevó a promover un amplio estudio diagnóstico y de proyecto de políticas públicas denominado "Sistemas Ambientales para la Planificación" en Venezuela, del que habría de surgir el primer Ministerio del Ambiente en 1978. Cuatro años antes, en octubre de 1974, el PNUMA había auspiciado una conferencia famosa en Cocoyoc (México) en donde se proclamó la llamada "Carta de Deberes y Derechos de los Estados" en donde se explicitaba que las políticas ambientales de todos los Estados deben promover y no afectar adversamente el actual y futuro potencial de desarrollo de los países en vías de desarrollo.

El resultado del surgimiento de este pensamiento crítico se fue diluyendo con sus propias estrategias prácticas. El énfasis por tratar de incorporar las problemáticas ambientales latinoamericanas dentro del concepto de desarrollo terminó en un fracaso rotundo: América del Sur desde la década del 70 terminó profundizando su intercambio ecológicamente desigual a través de la reprimarización de su economía ${ }^{13}$. El tratar de flexibilizar las instituciones para incorporar en las políticas públicas la "dimensión ambiental" terminó incorporando más problemas que soluciones. El Ecodesarrollo quedó atrapado en las mallas de la teoría de sistemas con la que buscaba reintegrar al sistema económico un conjunto de variables (crecimiento poblacional, cambio tecnológico) y de condiciones ambientales (procesos ecológicos, degradación ambiental). Esta visión sistémica y pragmática del Ecodesarrollo tenía una base teórica

\footnotetext{
12 Giglo, Nicolás. Estilos de desarrollo y medio ambiente en América Latina, un cuarto de siglo después. Santiago de Chile: CEPAL, 2006.

13 Peinado, Guillermo, Aín Mora, Bruno Ferrari, y Javier Ganem. «Las huellas de la contradicción entre desarrollo y ambiente. Un análisis del metabolismo socioeconómico en América del Sur a través de sus huellas ecológica e hídrica.» Revista del CESLA. International Latin American Studies Review(25), 2020: 3-22.
} 
muy endeble y reducía cualquier potencial de los saberes culturales y de los movimientos sociales por la apropiación de la naturaleza en la transparencia de las prácticas de la planificación ambiental ${ }^{14}$.

Las "estrategias del Ecodesarrollo" no alcanzaron a construirse totalmente que fueron hegemonizadas por un nuevo discurso más alineado con las políticas dominantes del momento. Este discurso basado en la noción de "desarrollo sostenible" se mantiene, en parte, hasta la fecha. Este concepto fue adoptado en un contexto caracterizado por la caída del keynesianismo y el ascenso del neoliberalismo que garantizaba nuevas reformas económicas en los países periféricos como la liberalización comercial y financiera, la privatización de las empresas y servicios públicos y un Estado que facilitaba fiscalmente las inversiones extranjeras. Este enfoque cambió la economía del desarrollo girando hacia una estrategia con base en el mercado liderada por los organismos internacionales de crédito como el Banco Mundial (BM) y el Fondo Monetario Internacional (FMI). Aquí el logro de "crecimiento económico sostenible" depende de la creación de mercados sin distorsiones y competitivo. Estos sucesos simbolizan el retorno de la economía del desarrollo a la ortodoxia neoliberal.

Bajo este marco histórico, el concepto de Desarrollo Sustentable fue expuesto en "Nuestro Futuro Común" en 1987 -o, como se lo conoce, el Informe Brundtland-, publicado por la Comisión Mundial sobre Medio Ambiente y Desarrollo a solicitud del Secretario General de las Naciones Unidas. La definición que se instaló y recorrió rápidamente por los discursos políticos de la época fue el entendimiento de que el desarrollo sostenible representaba el "proceso que permite satisfacer las necesidades de la población actual sin comprometer la capacidad de atender a las generaciones futuras".

El informe de Brundtland, revela como dice Escobar "la esencia de la actitud gerencial del planeta Tierra [...] en donde lo que está en juego es la continuidad de los modelos de crecimiento y desarrollo a través de estrategias administrativas adecuadas"15. La clave del discurso del "desarrollo sostenible" es dilucidar los

\footnotetext{
14 Leff, Enrique. «El retorno del orden simbólico: la capitalización de la naturaleza y las estrategias fatales del desarrollo sostenible.» En La racionalidad ambiental, de Enrique Leff, 88-132. México D.F.: Siglo XXI, 2004.
}

${ }^{15}$ Escobar, Arturo. La invención del Tercer Mundo. Caracas: El perro y la rana, 2007. pp. 324-325 
mecanismos adecuados que nos lleven a sacar el máximo de los recursos de la Tierra. El científico social continúa hablando en nombre de la Tierra sin permitir "que un campesino peruano, un nómada africano o un trabajador cauchero del Amazonas tenga algo que decir al respecto"16

Este "nuevo" discurso intentaba lograr una política de consenso disolviendo cualquier contradicción que pudiese haber entre sostenibilidad económica y sustentabilidad ambiental. Parafraseando a Leff, el discurso del desarrollo sustentable ya es ambivalente en la polisemia del término sustainability, que integra dos significados: el primero, traducible como sustentabilidad, implica la incorporación de las condiciones ecológicas del proceso económico; y el segundo, que se traduce como sostenibilidad, implica perdurabilidad en el tiempo del proceso económico. Es decir, que si la crisis ambiental es producto de la negación de las bases naturales en las que se sostiene el proceso económico, entonces la sustentabilidad ecológica aparece como condición de la sostenibilidad temporal del proceso económico. De esta manera, el discurso del desarrollo sostenible ha llegado a afirmar el propósito de hacer sostenible el crecimiento económico a través de los mecanismos del mercado, asignando valores económicos y derechos de propiedad a los recursos y servicios ambientales lo cual no ofrece una justificación rigurosa sobre la capacidad del sistema económico para incorporar las condiciones ecológicas y sociales ${ }^{17}$.

En el contexto de este discurso debe ser entendida la elaboración de la Agenda 21 en la Conferencia de las Naciones Unidas sobre Medio Ambiente y Desarrollo, celebrada en Río de Janeiro en junio de 1992. Esta agenda le da legitimidad y termina de disolver cualquier tipo de contradicción entre ambiente y desarrollo. Las políticas neoliberales en boga que planteaban como los mecanismos de mercado resuelven los problemas de asignación de recursos, también se muestran eficientes para hacer coincidir las condiciones ecológicas y los valores culturales con un proceso económico en crecimiento. Bajo este esquema de pensamiento, la crisis ambiental no es un problema de la acumulación de capital sino un problema de la mala asignación de los

\footnotetext{
16 Escobar, Arturo. La invención del Tercer Mundo. Caracas: El perro y la rana, 2007.

17 Leff, Enrique. «El retorno del orden simbólico: la capitalización de la naturaleza y las estrategias fatales del desarrollo sostenible.» En La racionalidad ambiental, de Enrique Leff, 88-132. México D.F.: Siglo XXI, 2004.
} 
derechos de propiedad y de pequeñas "fallas de mercados". Los economistas de Harvard comienzan a hablar del ambiente en estos términos y sus "soluciones" comenzaban a llegar: "Muéstrenme un recurso desperdiciado o un ambiente degradado y les mostraré un subsidio o una falla para establecerlas condiciones básicas que permitirían que el mercado funcionara eficientemente... Si tuviera que plantear la solución en una frase sería esta: Todos los recursos deberían tener propietarios, todas las gentes deberían tener derechos". 18

Esta racionalidad económica que conlleva a la reconciliación entre ambiente y crecimiento otorga un nuevo concepto de "medio ambiente", en dónde este se transforma como un recurso disponible. Este nuevo "ambiente" está íntimamente ligado a la visión de la naturaleza que tiene el sistema urbano industrial. Los materiales que circulan en este sistema productivo son materias primas, productos industriales, desechos tóxicos, "recursos". La "naturaleza es reducida a un ente estático, un mero apéndice del medio ambiente. Junto con el deterioro físico de la naturaleza, presenciamos su muerte simbólica". ${ }^{19}$

La Economía Ambiental, brazo "ecológico" de la economía neoclásica, establece que los derechos privados, las relaciones costo-beneficio y la asignación óptima de los recursos y de los sujetos de contaminación se resuelven mediante sistema de $\operatorname{precios}^{20}$. De esta manera solo unos pequeños ajustes al sistema de mercado pueden iniciar una era de "desarrollo ambientalmente benigno". Se cree que los problemas ambientales son, en esencia, problemas de administración económica.

En síntesis, la vieja creencia de que el desarrollo podía incorporar al ambiente y a los viejos problemas de nuestra región fue reemplazada por una noción que ni siquiera admitía que hubiese una contradicción entre ambiente y producción. Una de las razones puede haber sido la falta de elaboración teórica. Despegarse de la noción de desarrollo, tan imperante en la época no fue cosa fácil y menos aún para una disciplina que comenzaba a nacer.

\footnotetext{
${ }^{18}$ Escobar, Arturo. La invención del Tercer Mundo. Caracas: El perro y la rana, 2007.

${ }^{19}$ Escobar, Arturo. La invención del Tercer Mundo. Caracas: El perro y la rana, 2007.

20 Pengue, Walter Alberto. «La Economía Ecológica y el desarrollo en América Latina.» En Vertientes del pensamiento agroecológico: fundamentos y aplicaciones, de Miguel (Comp.) Altieri, 125-154. Medellín: Sociedad Cientifica Latinoamericana de Agroecología (SOCLA), 2009
} 
Por otro lado, hay que reconocer que la visión crítica de los "ecodesarrollistas" fue rápida y contundente. La mayoría de aquellos autores formarán parte del armazón teórico que va a luchar contra la idea hegemónica del "desarrollo sostenible"21. Si bien no es un corpus teórico acabado ni mucho menos preciso en sus delimitaciones tiene un alto componente de un pensamiento genuinamente latinoamericano que ayuda a pensar los problemas ecológicos de la región desde diferentes perspectivas.

\section{EL PROYECTO IDENTITARIO: EL ECOLOGISMO POPULAR, EL CAMINO EN EL QUE ANDAMOS, UN CAMINO CON IDEAS PROPIAS.}

Las nuevas ideas ambientalistas en nuestro continente no pueden entenderse sin el contexto de la emancipación de los pueblos indígenas como uno de los hechos políticos más relevantes del fin del siglo XX. Éstos han ido ganando espacios políticos para legitimar sus derechos culturales a sus territorios étnicos; a sus lenguas y costumbres; a su dignidad y autonomía. Allí se está gestando una nueva conciencia sobre los derechos de los pueblos indígenas a autogestionar los recursos naturales y el entorno ecológico.

Del proceso de globalización regido por la racionalidad económica y las leyes del mercado que predominaban en el discurso del "desarrollo sostenible" se emerge una política del lugar, del espacio y del tiempo movilizada por los nuevos derechos culturales de los pueblos, legitimando reglas más plurales y democráticas de convivencia social y de reapropiación de la naturaleza. En esta reafirmación de las identidades se manifiesta lo real de la naturaleza y lo verdadero de la cultura frente a una lógica económica que ha generado un proceso de degradación socioambiental que afecta las condiciones de sustentabilidad. El territorio se transforma en algo importante para las ideas ambientales: "El territorio es lugar porque allí arraiga una identidad en la que se enlazan lo real, lo imaginario y lo simbólico. El ser cultural elabora su identidad construyendo un territorio, haciéndolo su morada. Las geografías se vuelven verbo. Las culturas, al significar a la naturaleza con la palabra, la convierten en acto; al irla

\footnotetext{
${ }^{21}$ Entre otros se pueden nombrar a Iván Restrepo, Enrique Leff, Vicente Sánchez, Héctor Sejenovich, Hilda Herzer y Margarita Merino de Botero.
} 
nombrando, van construyendo territorialidades a través de prácticas culturales de apropiación y manejo de la naturaleza".22

Dentro de este embate contra las posiciones hegemónicas, dividiremos a las ideas ambientalistas latinoamericanas en dos grandes ejes: por un lado aquellas que se basan en un orgullo agroecológico y que están presentes, especialmente, en Mesoamérica y los Andes; en una segunda parte abordaremos al "ecologismo popular" y la ecología política latinoamericana que se fue formando al calor de una creciente conflictividad socioambiental que dio lugar a redes de activistas denunciando la extracción de recursos naturales y la vigencia de antiguas cosmovisiones indígenas.

\section{LA AGROECOLOGÍA LATINOAMERICANA: HACIA LA CONSTRUCCIÓN DE LO ALTERNATIVO.}

La naturaleza del pensamiento agroecológico surgió a finales de los años setenta como respuesta a las primeras manifestaciones de la crisis ecológica y social en el campo. En síntesis, se presentaba como una crítica a lo que se denominaba como "revolución verde", modelo de producción basado en el manejo químico e industrializado de los recursos naturales. En esos inicios esta disciplina no era teórica, sino que se expresaba a través de las distintas formas de resistencia practicadas por grupos campesinos e indígenas que generaron una dinámica de participativa que actuó como plataforma de enfrentamiento a la acción de las multinacionales. Estas manifestaciones de resistencia se negaban a aceptar los paquetes de agroquímicos, vinculados a las semillas híbridas que destruían su manejo tradicional, deterioraban sus bienes comunales y atentaban contra sus identidades socioculturales. ${ }^{23}$

Un ejemplo del orgullo agroecológico andino y mesoamericano es el Pratec en Perú formado por agrónomos disidentes de la escuela de La Molina. Reaccionaron en contra de su propia enseñanza y fueron críticos de la noción uniformizadora de "desarrollo" tan en boga en esa época. En la praxis, investigaron y aplicaron las epistemologías agrarias de la Sierra expresadas en técnicas agronómicas propias y en

\footnotetext{
22 Leff, Enrique. «El retorno del orden simbólico: la capitalización de la naturaleza y las estrategias fatales del desarrollo sostenible.» En La racionalidad ambiental, de Enrique Leff, 88-132. México D.F.: Siglo XXI, 2004.

${ }^{23}$ Sevilla Guzmán, Eduardo. "La participación en la construcción histórica latinoamericana de la Agroecología y sus niveles de territorialidad.» Política y Sociedad Vol. 52 N², 2015: pp. 351-370.
} 
la conservación de semillas de muchas variedades y especies a cargo de campesinas y campesinos indígenas. ${ }^{24}$

Por otro lado, estas ideas en el continente se desarrollaron a través de la práctica de Stephen Gliessman, que tras varios años trabajando en Costa Rica y México en la década de los setenta, tomó un puesto en la Universidad de Tabasco (México) y llevo las experiencias aprendidas durante su tiempo en América Central relacionadas con las prácticas agrícolas de los campesinos. Gliessman dictó en esa universidad el que, probablemente, fue el primer curso universitario de agroecología. En 1981, Gliessman se mudó a la Universidad de California y comenzó a construir un equipo de colegas y estudiantes que posteriormente han establecido vínculos duraderos con productores y comunidades agroecológicas, entre las que se encuentra la Red de Agroecología Comunitaria ${ }^{25}$.

Luego del auge del "desarrollo sostenible", muchos autores hicieron más visible el estudio de la agroecología centrándose en las propias prácticas agrícolas ancestrales y del respeto al conocimiento indígena. Este implicó un "redescubrimiento" de la Agroecología que la hegemonía del Ciencia Moderna había invisibilizado. Fue esta práctica pluriepistemológica, la cual a través de metodologías participativas produjo una "alternativa científica basada en la constatación empírica de que el conocimiento sobre el manejo de la naturaleza del pasado, e incluso el generado en las culturas marginadas por la civilización industrial, poseía los principios ecológicos que permitían evitar, no solo el deterioro medioambiental; sino también el social". ${ }^{26}$

Dentro de la influencia de los estudios y las prácticas agroecológicas contemporánea se puede citar al agrónomo Efraín Hernández Xolocotzi (1913-1991), cuya trayectoria desembocó en una nutrida y competente escuela de etno-ecólogos mexicanos entre los que se encuentra Víctor Toledo ${ }^{27}$. En ellos se inspira un movimiento campesino mexicano del siglo XXI que se basa en el refrán "Sin maíz no hay país". En los

\footnotetext{
${ }^{24}$ Martínez-Alier, Joan, Hector Sejenovich, y Michiel Baud. «El ambientalismo y ecologismo latinoamericano.» En Gobernanza ambiental en América Latina, de Fabio de Castro, Barbara Hogenboom y Michiel (Coordinadores) Baud, 39-73. Buenos Aires : Consejo Latinoamericano de Ciencias Sociales (CLACSO), 2015.

${ }^{25}$ Sevilla Guzmán, Eduardo, y Graham Woodgate. «Agroecología: fundamentos del pensamiento social agrario y teoría sociológica.» Agroecología Vol. 8 No2, 2013: 27-34.

${ }^{26}$ Sevilla Guzmán, Eduardo. "La participación en la construcción histórica latinoamericana de la Agroecología y sus niveles de territorialidad.» Política y Sociedad Vol. 52 No2, 2015: 351-370: pp. 354

${ }_{27}$ Toledo, Victor. «La agroecología en Latinoamerica: tres revoluciones, una misma transformación.» Agroecología N6, 2012: pp. $37-46$.
} 
escritos de Toledo la agricultura indígena es una de las principales fuentes del ecologismo latinoamericano.

Estos actores y movimientos agroecológicos han contribuido a la creación y al trabajo de numerosas instituciones como el Movimiento de los Trabajadores Sin Tierra (MST), el Movimiento Agroecológico Latinoamericano (MAELA) y la Sociedad Científica Latinoamericana de Agroecología (SOCLA), entre otras.

Ese rechazo al pensamiento occidental y la idea de que los pueblos cuya situación y prácticas son investigadas aportan sus propias perspectivas y conocimientos fue una idea que Robert Chambers de Sussex University desarrolló a partir de Paulo Freire ${ }^{28}$ y Orlando Fals Borda ${ }^{29}$, es decir, una idea latinoamericana. El tema de la colonialidad es abordado en numerosos autores contemporáneos y tiene utilidad para interpretar la crisis ambiental en términos de pérdida de conocimientos y culturas indígenas, lo que se denomina como "epistemicidios". Arturo Escobar y Gustavo Esteva son un ejemplo de dichos autores, pensadores destacados del postdesarrollismo que tienen raíces antiguas en el pensamiento de América (o AbyaYala).

En esta misma línea se pueden incluir autores como Héctor Alimonda y su crítica sobre la modernidad y la colonización de la naturaleza ${ }^{30}$ y Eduardo Gudynas y su crítica al neoextractivismo. Este último autor entiende al neoextractivismo como el estilo de desarrollo bajo los denominados "gobiernos progresistas" en América Latina cuyo centro económico es un entramado productivo escasamente diversificado y muy dependiente de una inserción internacional como proveedores de materias primas en donde el Estado juega un papel más activo en la redistribución de los excedentes

\footnotetext{
${ }^{28}$ Pedagogo brasilero (1921-1997), referente central de la denominada pedagogía de la liberación y crítico de los mecanismos educativos tradicionales o "educación bancaria" en donde el educador es el único poseedor de conocimientos y quien va transmitir los conocimientos a los educandos. Según Freire, esta pedagogía convierte el educando en un sujeto pasivo, y por tanto, en un sujeto oprimido. Este autor contrapone este tipo de educación con una pedagogía crítica que permita una participación activa en los educandos para promover el cambio radical de la sociedad en la que éste se sitúa.

${ }^{29}$ Sociólogo colombiano (1925-2008) y uno de los primeros autores en proponer la denominada descolonización de las ciencias sociales en América Latina analizando los efectos del colonialismo y la modernidad en esta región. Su metodología, influenciada por la teología de la liberación, une el pensamiento crítico con la investigación-participativa en los territorios.

30 Alimonda, Héctor. La naturaleza colonizada: Ecología política y minería en América Latina. Buenos Aires: CLACSO, 2011.
} 
generados por ese extractivismo, pero profundiza los impactos socioambientales negativos ${ }^{31}$.

Estos debates se dieron en parte en nuestro continente durante el siglo XXI. Por una parte, en Ecuador, el debate político tras 2007 introdujo palabras como Sumak Kawsay, "Buen Vivir", concepto que fue reelaborado en artículos y tesis por intelectuales quechuas como Carlos Eloy Viteri desde el año 2000. Este concepto se convirtió en objetivo nacional en la Constitución ecuatoriana del 2008. Algo similar ocurrió en la Constitución de Bolivia de 2009 con la expresión aymara, Suma Qamaña ${ }^{32,33}$.

Estas expresiones están sujetas a un amplio debate en la ecología política latinoamericana. Concretamente, podemos observar tres aristas conceptuales en estas denominaciones. En primer lugar existe un punto de vista culturalista (donde se destacan autores como Luis Macas y David Choquehuanca) que se centra en la cultura de los pueblos indígenas del Abya Yala, la dicotomía occidente-indigenismo y la opresión del "capitalismo occidental" a las formas de vida comunitarias. Por otra parte, se observa una arista más ecologista cuya problemática principal es la depredación del desarrollo capitalista a la madre tierra y la garantía de los derechos de la naturaleza. Aquí podemos citar autores como Alberto Acosta o Eduardo Gudynas. Por último, existe una vertiente ecomarxista liderada por Álvaro García Linera centrada en el estudio de las estructuras socioeconómicas y donde el buen vivir es un medio para terminar con la explotación de seres humanos por otros seres humanos y garantizar el "socialismo comunitario"34.

\section{El ECOLOGISMO POPULAR Y El NACIMIENTO DE LA ECOLOGÍA POLÍTICA LATINOAMERICANA}

\footnotetext{
${ }^{31}$ Gudynas, Eduardo. «Diez tesis urgentes sobre el neoextractivismo.» En Extractivismo, política y sociedad, de AA.VV, 187-225. Quito: CAAP CLAES, 2009.

32 Le Quang, Matthieu, y Tamia. Vercoutère. Ecosocialismo y Buen Vivir. Diálogo entre dos alternativas al capitalismo. Quito: Instituto de Altos Estudios Nacionales (IAEN), 2013

${ }^{33}$ Acosta, Alberto. «El Buen Vivir, una utopía por (re)construir.» CIP-Ecosocial - Boletín ECOS (CIP-Ecosocial - Boletín ECOS), 2010.

34 Le Quang, Matthieu, y Tamia. Vercoutère. Ecosocialismo y Buen Vivir. Diálogo entre dos alternativas al capitalismo. Quito: Instituto de Altos Estudios Nacionales (IAEN), 2013
} 
El ecologismo popular fue influenciado por las nuevas ideas de la Teología de la Liberación $^{35}$ y los diversos movimientos sociales en la región. Se convirtió en una corriente crítica de los modelos que propugnaban por un mayor crecimiento económico a través de los modelos impuestos por los países industrializados. Este concepto desarrollado por el catalán Joan Martínez Alier, abarca movimientos heterogéneos que son a veces "puramente reactivos y que en general no aspiran a lograr influencia política per se, sino que emergen como una reacción a problemas ambientales específicos". ${ }^{36}$ Martínez Alier define al ecologismo popular como la expresión de una "economía moral" que se enfrenta a la mercantilización citada por Leff y se manifiesta en las fronteras extractivas. Las poblaciones campesinas y/o indígenas protestan contra las industrias extractivas de minerales o de biomasa, usando distintos lenguajes de valoración ${ }^{37}$.

Este ecologismo popular latinoamericano tiene su centro en redes de información y como las de Observatorio de Conflictos Mineros en América Latina (OCMAL) y el Observatorio Latinoamericano de Conflictos Mineros (OLCA), centradas en Chile. Ya que es internacional tiene conexiones con movimientos de resistencia en la India y en África, y también tiene coincidencias con el movimiento por la justicia ambiental en Estados Unidos. El ecologismo se hizo presente en numerosos conflictos locales en las últimas décadas. Incluso, se crearon Atlas y mapas que muestran estos $\operatorname{conflictos}^{38}$.

Este movimiento ecologista tiene elaboraciones teóricas propias y un vocabulario específico. En materia económica se basa en la economía ecológica, rama antagónica de la economía ambiental. La economía ecológica aborda la interrelación entre Economía y Naturaleza de forma diferente, entendiendo la Economía como un sistema abierto en constante interacción con los sistemas físico y social. Más allá de los

\footnotetext{
35 Uno de los máximos referentes es Leonardo Boff. Este teólogo brasilero conectó las ideas de la teología de la liberación que inspiraron a los sacerdotes tercermundistas con las ideas ecologistas y la denuncia al modelo de depredación que brindaba el capitalismo en América Latina.

${ }^{36}$ Martínez-Alier, Joan, Hector Sejenovich, y Michiel Baud. «El ambientalismo y ecologismo latinoamericano.» En Gobernanza ambiental en América Latina, de Fabio de Castro, Barbara Hogenboom y Michiel (Coordinadores) Baud, 39-73. Buenos Aires : Consejo Latinoamericano de Ciencias Sociales (CLACSO), 2015.

pp. 59.

${ }^{37}$ Martínez-Alier, Joan, Héctor Sejenovich, y Michiel Baud. «El ambientalismo y ecologismo latinoamericano.» En Gobernanza ambiental en América Latina, de Fabio de Castro, Bárbara Hogenboom y Michiel Baud, 39-73. Buenos Aires: Consejo Latinoamericano de Ciencias Sociales (CLACSO), 2015.

38 Ver, por ejemplo, https:/lejatlas.org/
} 
valores monetarios de mercado que se aplicaba con el "desarrollo sustentable", se pretende también la evaluación física de los impactos ambientales de la economía humana, teniendo en cuenta indicadores físicos sobre flujos de energía, ciclos de materias primas, entre otros. De esta forma se tiene en cuenta aspectos que tradicionalmente se dejaban de lado como son la disponibilidad de recursos y energía y la generación de residuos (materiales y energía degradados). Por otra parte, la economía ecológica es multidisciplinaria y entiende que los problemas ambientales van ligados al mundo de la política y de las relaciones de poder y, por tanto, no pueden estudiarse al margen de estas cuestiones. La actitud más general dentro de la economía ecológica es la de rechazo radical de todo el marco analítico de la economía neoclásica hegemónica. 39

Siguiendo con la idea de conformación de un diccionario propio, los intelectuales que estudian el ecologismo popular y los movimientos que de él surgen utilizan el término "deuda ecológica" para oponerse a la pérdida de la capa de ozono y al cambio climático. Bajo esta consigna también se estudian cuestiones derivadas del intercambio ecológico desigual, término que combina las nuevas teorías de la dependencia y la cuestión ecológica. A través de esta teoría, se plantea el hecho de que un patrón de comercio internacional aunque fuera financieramente equilibrado puede ser ecológicamente desigual por medio de un desbalance del contenido de recursos naturales (en términos de materiales y energía) de las exportaciones netas ${ }^{40}$.

Otras palabras del nuevo vocabulario están asociadas a las nociones de justicia ambiental que reclaman un tribunal penal internacional en materia ambiental y una convención internacional sobre "ecocidio". Otro de los términos más utilizados por el movimiento de justicia ambiental es el de "biopiratería", introducido en 1993 por Pat Mooney y muy difundido mundialmente por Vandana Shiva. En América Latina se destaca la Red de Acción por la Biodiversidad que coordina Carlos Vicente, activista y autor de libros sobre el tema.

\footnotetext{
39 Ramos Gorostiza, José Luis. «Medio natural y pensamiento económico: historia de un reencuentro.» Principios. Revista N², 2005: pp. 47-70

${ }^{40}$ Belloni, Paula, y Guillermo Peinado. «Inserción externa, capitales trasnacionales e intercambio ecológicamente desigual en la América del Sur posneoliberal.» Sociedad y Economía No25, 2013: 15-38.
} 
Siempre bajo esta pluralidad de pensamientos y teorías, Enrique Leff en Ecología y Capital en 1986 y el economista James O'Connor (conocido autor que elabora la "Segunda contradicción del Capitalismo") explicaron que los crecientes costos sociales y ambientales que causa el (mal contado) crecimiento de la economía, son la causa de la explosión de protestas ecologistas. Esto tiene su correlato en la realidad concreta. Actualmente hay un proceso en el mundo en donde se expulsa a los indígenas y campesinos de sus tierras, se expropia de manglares por la industria camaronera, de acaparamiento de tierras para plantaciones de árboles o agro-combustibles, para la explotación de minerales y represas, a cargo de empresas multinacionales o estatales, procesos neocoloniales de apropiación de recursos naturales y territorios donde aparecen actores nuevos como las empresas chinas.

\section{CONCLUSIONES: LA CUESTIÓN AMBIENTAL COMO CENTRO DE LOS DEBATES SOCIALES.}

En el trabajo nos hemos propuesto reconstruir algunas "corrientes" del pensamiento latinoamericano ambientalista. Bajo el paraguas teórico que nos brinda Devés Valdés pudimos encontrar dos caminos antagónicos y diferentes en la breve historia de las ideas ambientalistas latinoamericanas.

En un principio, bajo la influencia de la problemática ambiental de los países "desarrollados" se elaboró una propuesta que, si bien intentaba ser identitaria y resolver las problemáticas específicas de nuestra región, terminó por deformarse y convertirse en una idea modernizadora. Esa propuesta era el "Ecodesarrollo", que intentaba romper con el enfoque de "Los límites al Crecimiento" del Informe Meadows de 1972. Si bien esta "corriente" incorporaba las cuestiones ambientales y sociales que acuciaban a los países de América Latina, el resultado no fue favorable y se fue diluyendo con las propias estrategias prácticas. Tal como dijo Leff, el tratar de flexibilizar las instituciones para incorporar en las políticas públicas la "dimensión ambiental" terminó incorporando más problemas que soluciones. Esta visión sistémica y pragmática tenía una base teórica muy endeble y reducía cualquier potencial de los saberes culturales y de los movimientos sociales por la apropiación de la naturaleza en la transparencia de las prácticas de la planificación ambiental. 
Este pensamiento que buscaba ser propio terminó atrapado y fue hegemonizado por un nuevo discurso basado en las nociones de "desarrollo sostenible" que buscaba garantizar el crecimiento económico teniendo en cuenta lo ambiental bajo mecanismos del mercado, asignando valores económicos y derechos de propiedad a los recursos y servicios ambientales. Esta idea construida en el Informe Brutland elimina cualquier contradicción entre ambiente y producción y crea un tipo de "desarrollo" que puede ser aplicado en cualquier punto geográfico.

Este movimiento modernizador, que busca homogeneizar las problemáticas ambientales y sociales mundiales, fue contrapuesto por un movimiento que continúa pensándose y elaborándose a partir de ideas latinoamericanas. Destacamos las prácticas del Pratec en Perú; las ideas fundadoras de Stephen Gliessman; la influencia de los estudios y prácticas de Xolocotzi y más tarde de Toledo; y, más recientemente, el abordaje a temas de colonialidad y políticas del "Buen Vivir", entre los que citamos a Escobar y Esteva entre muchos otros.

Asimismo, las crecientes conflictividades en torno a la cultura del extractivismo y el esquema exportador de materias primas deriva en la formación de una nueva corriente a la que denominamos "ecologismo popular" muy propia de América Latina y compartida con África, la India y el Asia sudoriental. El ecologismo popular y sus respectivos movimientos sociales han obligado a una actualización de una gran producción intelectual. Esta producción ha creado un vocabulario propio ("deuda ecológica", "biopiratería", "intercambio ecológico desigual", etc.) y se compone de un conjunto heterogéneos de distintas disciplinas que buscan abordar las cuestiones ambientales en América Latina.

Una disciplina que busca abordar todas esas aristas es la Ecología Política. En esta se despliegan acciones y se construyen pensamientos para relanzar al mundo hacia el re-enlazamiento de la naturaleza y la sociedad. Leff sostiene que en el territorio de la Ecología Política se construye desde la teoría, el discurso y la acción una variedad de ambientalismos en los que el pensamiento y los movimientos socioambientales de la región ocupan un papel preponderante. 
Dentro de esa Ecología Política y de forma complementaria aparece la Economía Ecológica, como una disciplina que ayuda a pensar la contradicción ambiente-producción desde otra óptica. Su principal característica es su carácter transdisciplinario que demanda la participación no sólo de economistas, sino también de cientistas naturales y otras disciplinas, lo que refleja fielmente el carácter multidisciplinar que la problemática ambiental exige.

Para concluir, coincidimos con Martínez Alier en que "el post-extractivismo es intelectualmente poderoso pero políticamente débil todavía" ${ }^{41}$. Si bien trata de abrirse paso con propuestas concretas de gobernanza continental e internacional y formas alternativas al desarrollo tradicional, su peso es débil. Sin embargo, en el análisis de las ideas en curso, el nuevo ciclo identitario constituye un poderoso elemento que puede hacer converger las distintas teorías críticas que se encuentran dislocadas. Estas ideas en su conjunto son una valiosa alternativa de resistencia contra el neoextractivismo hegemónico. Es aquí donde el ecologismo juega un papel crítico preponderante en los debates latinoamericanos actuales y futuros.

En este sentido, resulta de extrema necesidad incorporar a estos nuevos léxicos los saberes producidos localmente por los movimientos sociales. En este nuevo milenio, estos movimientos que emergen en los países latinoamericanos ${ }^{42}$ van incorporando la problemática ambiental y la lucha contra el neoextractivismo. Colocarlos en el centro de la escena (con sus avances y retrocesos) sin caer en un romanticismo esencialista que oponga tradición y desarrollo es esencial para poder garantizar un puente con lo mejor de nuestras ideas identitarias (Teología de la Liberación, Ecologismo Popular, Pedagogía crítica, Investigación Acción-Participativa, Teoría(s) de la Dependencia y Economía Popular, entre otras). El desafío es, entonces, incorporar en el pensamiento ambientalista latinoamericano toda esta teoría de la praxis sin caer en salidas homogéneas y unitarias para el continente y entendiendo que estos movimientos, si bien son locales o regionales, se inscriben en redes globales debido a su capacidad para

\footnotetext{
${ }^{41}$ Martínez-Alier, Joan, Hector Sejenovich, y Michiel Baud. «El ambientalismo y ecologismo latinoamericano.» En Gobernanza ambiental en América Latina, de Fabio de Castro, Barbara Hogenboom y Michiel (Coordinadores) Baud, 39-73. Buenos Aires : Consejo Latinoamericano de Ciencias Sociales (CLACSO), 2015.

${ }^{42}$ Aquí hablamos, por ejemplo, de las organizaciones campesinas ecuatorianas, el Movimiento de Trabajadores Rurales (MST) en Brasil, el Ejercito Zapatista de Liberación Nacional (EZLN) en México, la Vía Campesina en América Latina, los Ecofeminismos de la cuarta ola, entre muchísimos otros.
} 
conectarse en complejos y dinámicos circuitos que van de lo local a lo global y viceversa ${ }^{43}$.

\section{REFERENCIAS}

Acosta, Alberto. «El Buen Vivir, una utopía por (re)construir.» CIP-Ecosocial - Boletín ECOS (CIP-Ecosocial - Boletín ECOS), 2010.

Alimonda, Hector. La naturaleza colonizada: Ecología política y minería en América Latina. Buenos Aires: CLACSO, 2011.

Belloni, Paula, y Guillermo Peinado. «Inserción externa, capitales trasnacionales e intercambio ecológicamente desigual en la América del Sur posneoliberal.» Sociedad y Economía No25, 2013: 15-38.

Devés Valdes, Eduardo. «El pensamiento latinoamericano a comienzos del siglo XX: La reivindicación de la identidad.» CUYO, Anuario de Filosofía Argentina y Americana, $\mathrm{N}^{0}$ 14, 1997: 11-75.

Escobar, Arturo. La invención del Tercer Mundo. Caracas: El perro y la rana, 2007.

Estenssoro Saavedra, Fernando. «El ecodesarrollo como concepto precursor del desarrollo sustentable y su influencia en América Latina.» Universum, Universidad de Talca. Vol. 30. Nº 1, 2015: 81-99.

-. Historia del debate ambiental en la politica mundial (1945-1992). Santiago de Chile: Instituto de Estudios Avanzados. Universidad Santiago de Chile. , 2014.

Flórez Flórez, Juliana. «Los movimientos sociales y la crisis del desarrollismo.» CLACSO, 2008: 1-22.

Foladori, Guillermo. «La Economía Ecológica.» En ¿Sustentabilidad? Desacuerdos sobre el desarrollo sustentable, de Guillermo Foladori y Naina Pierri, 189-197. México D.F.: Universidad Autónoma de Zacatecas, 2005.

Giglo, Nicolás. Estilos de desarrollo y medio ambiente en América Latina, un cuarto de siglo después. Santiago de Chile: CEPAL, 2006.

Gudynas, Eduardo. «Diez tesis urgentes sobre el neoextractivismo.» En Extractivismo, politica y sociedad, de AA.VV, 187-225. Quito: CAAP - CLAES , 2009.

43 Flórez Flórez, Juliana. «Los movimientos sociales y la crisis del desarrollismo.» CLACSO, 2008: 1-22. 
Guimaraes, Roberto. «El discreto encanto de la cumbre de la tierra. Evaluación impresionista de Río-92.» Nueva Sociedad N²122, 1992: 86-103.

Le Quang, Matthieu, y Tamia. Vercoutère. Ecosocialismo y Buen Vivir. Diálogo entre dos alternativas al capitalismo. Quito: Instituto de Altos Estudios Nacionales (IAEN), 2013.

Leff, Enrique. «El retorno del orden simbólico: la capitalización de la naturaleza y las estrategias fatales del desarrollo sostenible.» En La racionalidad ambiental, de Enrique Leff, 88-132. México D.F.: Siglo XXI, 2004.

Martínez-Alier, Joan, Hector Sejenovich, y Michiel Baud. «El ambientalismo y ecologismo latinoamericano.» En Gobernanza ambiental en América Latina, de Fabio de Castro, Barbara Hogenboom y Michiel (Coordinadores) Baud, 39-73. Buenos Aires : Consejo Latinoamericano de Ciencias Sociales (CLACSO), 2015.

Peinado, Guillermo, Aín Mora, Bruno Ferrari, y Javier Ganem. «Las huellas de la contradicción entre desarrollo y ambiente. Un análisis del metabolismo socioeconómico en América del Sur a través de sus huellas ecológica e hídrica.» Revista del CESLA. International Latin American Studies Review(25), 2020: 3-22.

Pengue, Walter Alberto. «La Economía Ecológica y el desarrollo en América Latina.» En Vertientes del pensamiento agroecológico: fundamentos y aplicaciones, de Miguel (Comp.) Altieri, 125-154. Medellín: Sociedad Cientifica Latinoamericana de Agroecología (SOCLA), 2009.

Ramos Gorostiza, José Luis. «Medio natural y pensamiento económico: historia de un reencuentro.» Principios. Revista No2, 2005: 47-70.

Sejenovich, H, B Slutsky, y S Cabrera. Rescatando la historia perdida. El pensamiento ambiental latinoamericano a la luz de las contradicciones actuales del desarrollo. Buenos Aires: ENGOV, 2012.

Sevilla Guzmán, Eduardo. «La participación en la construcción histórica latinoamericana de la Agroecología y sus niveles de territorialidad.» Política y Sociedad Vol. $52 \mathrm{~N}^{0} 2,2015:$ 351-370.

Sevilla Guzmán, Eduardo, y Graham Woodgate. «Agroecología: fundamentos del pensamiento social agrario y teoría sociológica.» Agroecología Vol. 8 N² 2, 2013: 27-34.

Toledo, Victor. «La agroecología en Latinoamerica: tres revoluciones, una misma transformación.» Agroecología Nº6, 2012: 37-46. 


\title{
Ideas About Development in the History of Latin American Environmental Thought
}

\begin{abstract}
This document aims to indentify, the different ideas on the relationship between production and the environment throughout recent history in Latin America. Specifically, we pretend to elaborate how these ideas between production and the environment penetrated civil society and how their relationship was around the concept of development. The objective of this work is not to generate consensus on conversions and the main contributions to Latin American environmental thought. On the conrtary, our elaboration seeks to provide a general and personal vision, whose intention is to mark some critical axes that require further development of this thinking in our region. In relation to the general objective, it is verified that the historical memory of environmental ideas derived from two antagonistic currents. On one hand, a proposal associated with sustainable development expressed by international organizations where there is a reconciliation between development and the environment. Conversely, a more identity idea is presented that points out the contradictions between the concept of development and environment and proposes the elaboration of alternative economic practices that face traditional hegemonic visions of sustainable development.
\end{abstract}

Keywords: environmentalist thought; ecodevelopment; sustainable development; popular ecology.

Recibido: 04/10/2020

Aprobado: 09/11/2020 